\title{
Photonic Choke-Joints for Dual-Polarization Waveguides
}

\author{
Edward J. Wollack, Kongpop U-yen, and David T. Chuss \\ NASA Goddard Space Flight Center, Greenbelt, Maryland, 20771, USA
}

\begin{abstract}
Photonic choke joint (PCJ) structures for dualpolarization waveguides have been investigated for use in device and component packaging. This interface enables the realization of a high performance non-contacting waveguide joint without degrading the in-band signal propagation properties. The choke properties of two tiling approaches, symmetric square Cartesian and octagonal quasi-crystal lattices of metallic posts, are explored and optimal PCJ design parameters are presented. For each of these schemes, the experimental results for structures with finite tilings demonstrate near ideal transmission and reflection performance over a full waveguide band.
\end{abstract}

Index Terms - Antenna feeds, periodic structures, waveguide mounts, component packaging, waveguides.

\section{INTRODUCTION}

A waveguide joint provides an interface for a variety of modularized waveguide components. Historically, both cover and choke flanges have been used [1] to provide a low impedance ohmic contact for waveguide connection and splitblock component packaging. The cover flange has a flat smooth surface that can be aligned with sufficient accuracy to produce minimal discontinuity and loss. The choke flange uses impedance transformation techniques to provide a virtual electrical short at the waveguide wall. This avoids voltage breakdown at the waveguide joint for limited-bandwidth highpower applications. By design, the physical contact provides a high thermal and electrical conductance interface.

To achieve high thermal isolation, photonic crystal structures have been used as an alternative approach for realizing waveguide joints [2]. Such a structure essentially forms an artificial dielectric [3] in the plane of the waveguide joint with a stop band. Conceptually, this can be realized by placing an infinite two-dimensional array of dielectric or metallic pillars perpendicular to the waveguide similar to the example shown in Fig 1(a). In practice, the allowable leakage sets the size of the finite array required. The joint's periodic structure is patterned on one side of the flange. The other side of the interface is flat and approximates a perfect-electric wall. The frequency response of the joint will depend on the shape of, the spacing between pillars and the gap between the pillar and the flat flange. These parameters can be adjusted to provide a virtual short at the edge of the waveguide without requiring physical contact between the two flange surfaces. Such a structure is highly suitable for low-power, noncontacting waveguide interfaces. It also can be used to provide thermal isolation, reduce mating tolerance between two waveguides, and simplify the waveguide assembly [4]. The space between the flanges can also be used to define a suitable enclosure for planar circuits $[5,6]$.

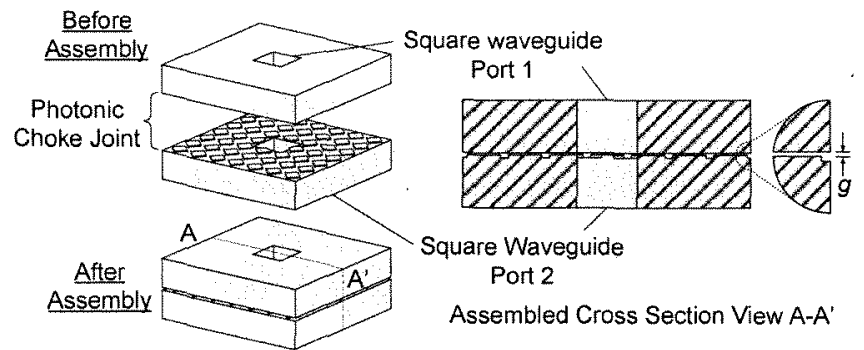

(a)

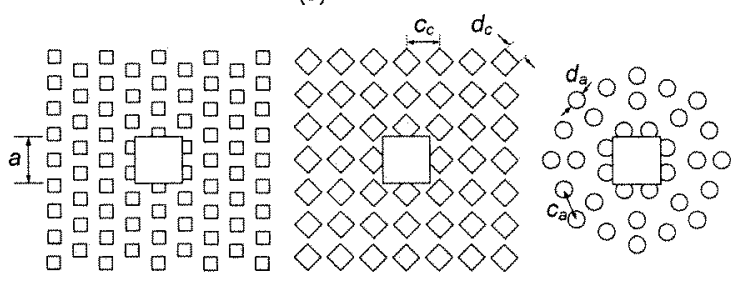

(b)

(c)

(d)

Fig. 1. (a) A 3-D model of the square waveguide with photonic choke joint and its cross-sectional view is presented. The plan views of pillar layout with finite (b) hexagonal, (c) Cartesian, and (d) octagonal tilings are shown.

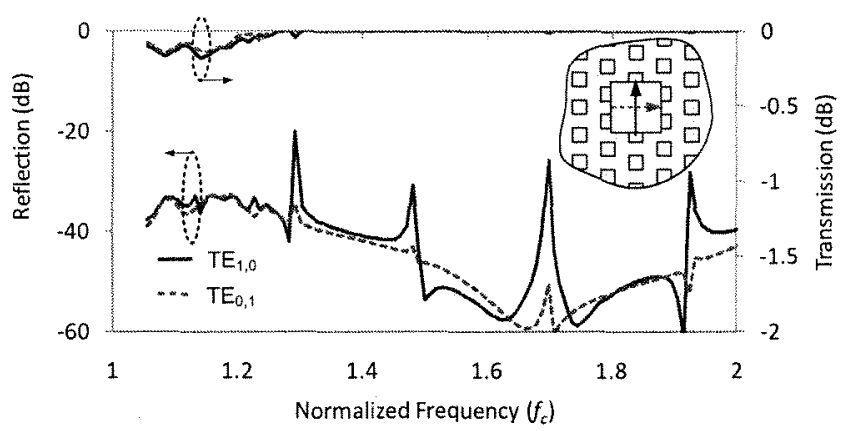

Fig. 2. The reflection and transmission responses are shown for a square waveguide joint employing a PCJ with hexagonal tiling (Fig. 1(b)) when excited in $\mathrm{TE}_{1,0}$ (solid) and $\mathrm{TE}_{0,1}$ (dashed) mode. The simulated flange spacing $(g)$ is $0.0088 \mathrm{a}$.

In previous work [2], a photonic choke joint (PCJ) has been demonstrated for rectangular waveguide with a hexagonal tiling of metallic square pillars. Its tiling pattern is similar to the one shown in Fig. 1(b). In directly applying this approach to a square waveguide, the input return losses in the $\mathrm{TE}_{1,0}$ and $\mathrm{TE}_{0,1}$ modes are asymmetric, and in-band spurious responses are observed. For an example see Fig 2. In this figure, the frequency response is normalized to the waveguide cut-off frequency, $f_{c}=c / 2 a$, where $c$ is the speed of light in free space and $a$ is the width of the waveguide broad-wall. This behavior is a result of the two polarizations encountering different boundary conditions. Motivated by this observation we 
explore the use of rotationally-symmetric-tilings to eliminate these spurious responses.

In this paper, we demonstrate two PCJs suitable for use with dual polarization square waveguide. The Cartesian and octagonal tiling patterns are introduced in Section II. The optimal arrangement and dimensions of square pillars for the Cartesian tiling are described in Section III. Section IV describes the optimal arrangement of the circular pillars in the octagonally tiled structure. The performance as a function of the number of pillar rows is discussed in Section V. A comparison of measurements and simulations is provided in Section VI. Finally, conclusions are provided in Section VII.

\section{Photonic Choke JoINT GeOMETRIES}

We have considered two PCJ tiling arrangements that are highly suitable for waveguide implementation in terms of ease of fabrication and design. For fabrication simplicity, we use metallic pillars with either square or circular cross-sections arranged in four-fold and eight-fold rotationally symmetric patterns, respectively. This selection leads to the design of periodic Cartesian and octagonal PCJ tilings as shown in Fig 1 (c) and 1(d), respectively. The flange parallelism and gap spacing $(g)$ are controlled. Several waveguide PCJ parameters were studied to obtain the minimum power leakage over a large bandwidth. By scaling the optimal dimensions by the guide cutoff frequency this design approach can be applied to other dual-polarization guided wave structures [7]. Although we have also investigated the use of this technique for circular guide we present only the results for square cross-section guides in the interest of brevity.

\section{CARTESIAN TILING OF SQUARE PILLARS}

In this scheme, the orientation of the pillars is studied to determine maximum power confinement with respect to the laterally propagating waves. In the square waveguide case, the majority of the constituent modes propagate in the direction perpendicular to the waveguide walls. To study this effect, we constructed and simulated simple PCJ models using Ansoft HFSS software with five rows of pillars as shown in Fig. 3(a), (b) and (c). Perfect magnetic sidewalls are placed on two edges of the model to simulate an infinitely wide PCJ area. Perfect electric conductors are used on the upper and lower boundaries that define the PCJ tiling geometry. The input and output ports are excited by a plane wave. We find the structure's input impedance is a good indicator of the arrangements effectiveness. This impedance has only a reactive component since the structure has essentially no ohmic loss. For maximum field confinement, the input impedance should be as low as possible compared to the characteristic impedance of free space. This results in a wide stop band. We tuned the pillar width $\left(d_{c}\right)$ and the spacing between pillars $\left(c_{c}\right)$ of each arrangement relative to $a$. We

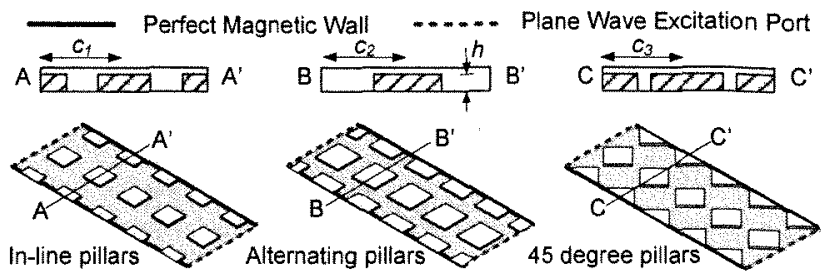

(a)

(b)

(c)

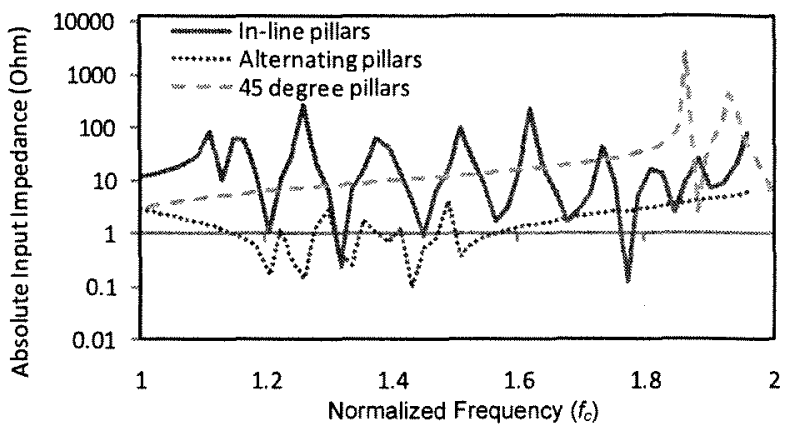

Fig. 3. The Cartesian tiling (a) in-line pillars, (b) alternating pillars and (c) pillars rotated by 45 degrees where the square pillar widths are $0.6 a, 0.75 a$ and $0.4 a$, respectively are shown. The pillar spacing's are $c_{1}=1.12 \mathrm{a}, c_{2}=1.12 \mathrm{a}$ and $c_{3}=0.68 \mathrm{a}$, respectively. The simulated flange spacing $g$ is $0.0088 a$.

observe that the tiling with in-line pillars produces the highest input impedance with numerous in-band spurious responses whereas the tiling with alternating pillars produces the lowest input impedance. We have selected the tiling with square pillars that are rotated by 45 degrees with respect to the orientation of the square waveguide. This arrangement satisfies the desired symmetry and removes the spurious responses (see Fig. 3). In all cases, the pillar height $h$ and flange spacing $g$ has an insignificant effect on the input impedance response [2] and they are set to $0.037 a$ and $0.0088 a$, respectively.

With proper tiling of the square pillars, we constructed the PCJ for square waveguide. The simulation results of the proposed PCJ with three rows are shown in Fig. 4 . The results show that the structure produces a leakage less than 0.001 up to $1.61 f_{c}$ when the flange spacing is below $0.0088 a$. The power leakage is determined through the S-parameter relationship: 1$\left|S_{21}\right|^{2}-\left|S_{11}\right|^{2}$. In addition, the Cartesian PCJ results in reflections less than $-24 \mathrm{~dB}$ without in-band spurious response up to $1.82 f$. The power leakage of less than $1 \%$ can be maintained when the gap spacing is less than $0.035 a$.

\section{OCTAGONAL TILING OF CIRCULAR PILLARS}

For a second realization of the PCJ, we used a quasi-crystal pillar arrangement with an eight-fold rotational symmetry [8]. The waveguide is placed at the center of this pattern as shown in dashed-lines in Fig 5. Three rows of circular pillars are placed at the vertices of the quasi-crystal arrangement. The optimized dimension of this PCJ yields the waveguide 


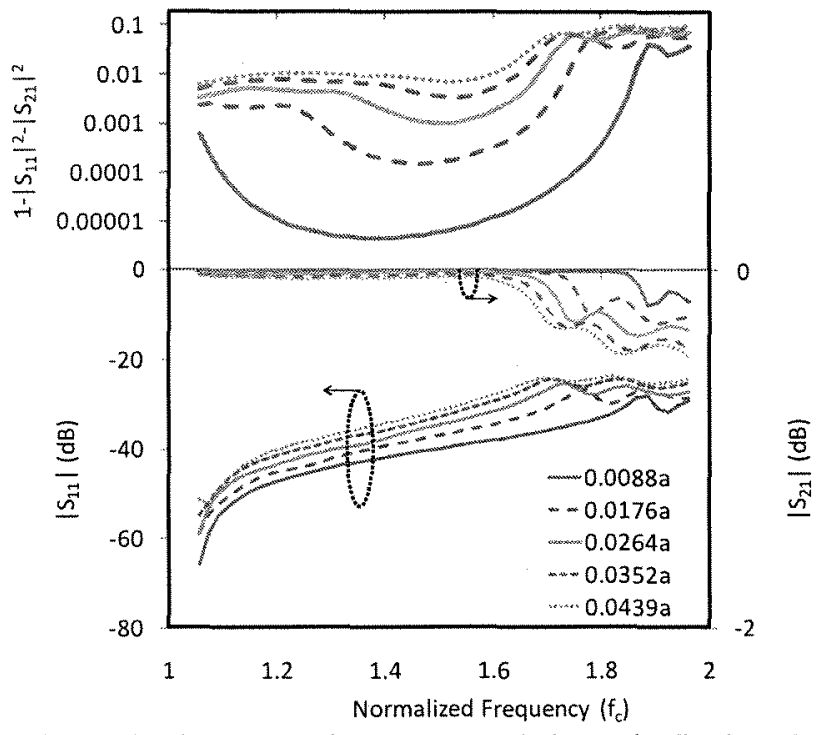

Fig. 4. The simulated power leakage, transmission and reflection of the Cartesian tiling are shown. This $\mathrm{PCJ}$ has three rows of square pillars with dimensions $c_{c}=0.68 a$ and $d_{c}=0.4 a$ for selected $g$ ranging from $0.0088 a$ to $0.0439 a$.

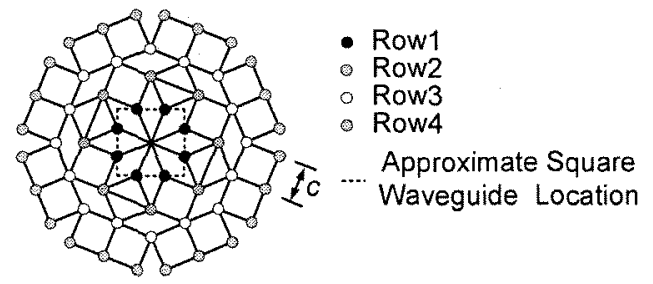

Fig. 5. The quasi-crystal tiling used for octagonal PCJ is shown. The dots indicate the center position of the cylindrical pillars.

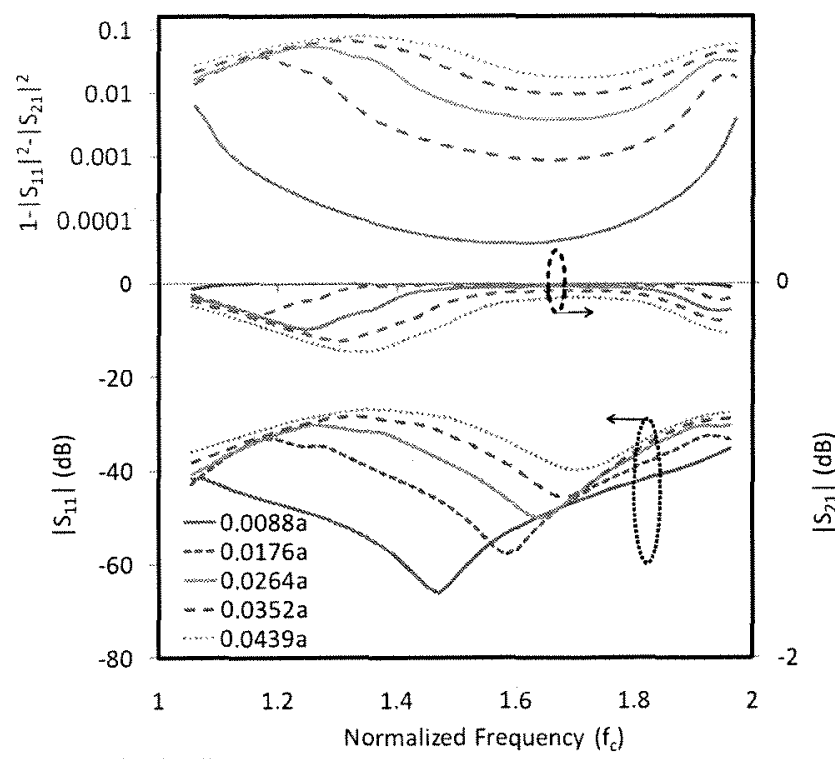

Fig. 6. The simulated power leakage, transmission and reflection of the octagonal tiling are shown. This PCJ has three rows of square pillars with dimensions $c_{c}=0.68 a$ and $d_{c}=0.18 a$ for selected $g$ ranging from $0.0088 a$ to $0.0439 a$. transition with low power leakage when the flange spacing is below $0.0088 a$ as shown in Fig. 6. This design results in reflections below $-26 \mathrm{~dB}$, up to $\sim 2 f_{c}$. From these results, it is clear that the PCJ with octagonal tiling pillars has higher leakage than that of the Cartesian tiling, and it is more sensitive to the flange-to-pillar gap spacing. Addition of another row of pillars reduces these concerns.

\section{NUMBER OF ROWS OF PILLARS IN WAVEGUIDE PCI}

The power leakage in the waveguide $\mathrm{PCJ}$ is dependent on the number of rows of pillars. As the number of row increases, the power leakage is significantly reduced around the center of the operating band. However, increasing the number of rows has little effect near the upper or lower end of the operating bandwidth as shown in Fig. 7 for PCJs with both Cartesian and octagonal tilings. Therefore for practical realizations, a finite number of rows of pillars can be used while maintaining a low loss interface.

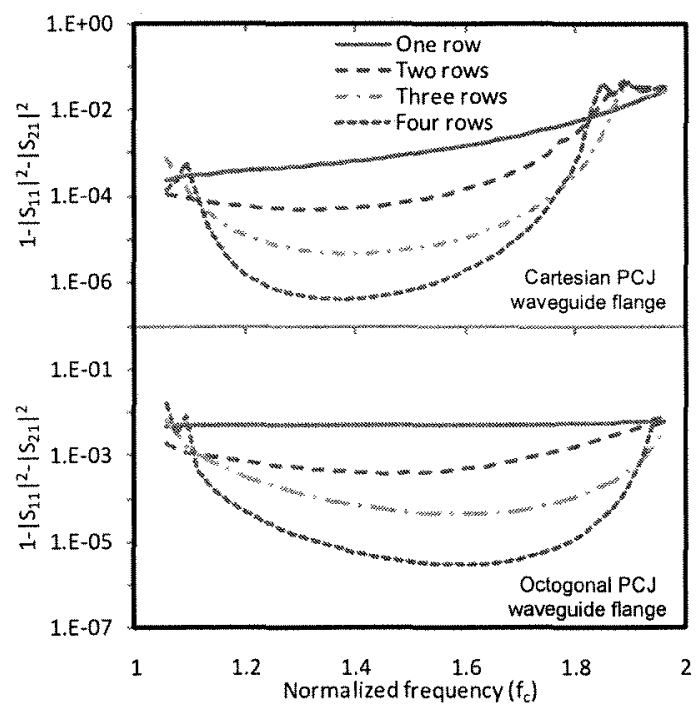

Fig. 7. The frequency response of the power leakage of the Cartesianand the octogonal-tiling waveguide photonic choke joints with different number of rows of pillars are shown.

\section{EXPERIMENTAL RESULTS}

Waveguide PCJ test fixtures were fabricated from copper. (See Fig. 8.) The designs are based on WR22.4 waveguide standard $\left(f_{c}=26.35 \mathrm{GHz}\right)$ and they were tested from 28 to 47 $\mathrm{GHz}$ with a HP-8510 network analyzer. The waveguide PCJs were connected to full-band rectangular-to-square waveguide transitions. A Thru-Reflect-Line calibration was performed in the square guide with the measurement reference planes 25.4 $\mathrm{mm}$ from the plane of the PCJ. The measured data were not corrected for the loss associated with these square waveguide extensions $(<0.04 \mathrm{~dB}$ loss). The return loss and transmission of the waveguide were measured at different gaps between the pillars and opposing flange ranging from $0 \mathrm{~mm}$ to $0.16 \mathrm{~mm}$ 


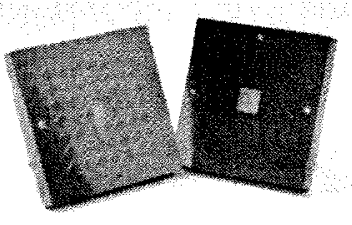

(a)

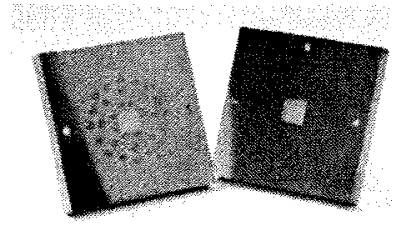

(b)
Fig. 8. The fabricated waveguide flanges with (a) Cartesian and (b) octagonal tiling photonic choke joint are shown.

$(0.028 a)$. The experimental results are in agreement with simulation. At zero gap spacing we observe constant loss across the measurement band consistent with the estimated loss of the waveguide extensions. As the gap size is increased, the high frequency response is degraded. This trend was observed both in our simulations and measurements. However, larger deviations and sensitivity to alignment were observed with the PCJ test samples. This can be seen in the high frequency response shown in Figures 9 and 10. Both PCJ designs provide low in-band power leakage of less than $3 \%$ when the gap size is smaller than $0.16 \mathrm{~mm}(0.028 a)$.

\section{CONCLUSION}

New PCJ structures with symmetrical tilings for dual polarization waveguides have been described. These high performance non-contacting waveguide joints are useful for providing a suitable working volume for planar circuitry, high thermal isolation, and DC blocking in a relatively small volume. These designs exhibit low loss and broadband symmetric polarization response.

\section{REFERENCES}

[1] G. Montgomery, R.H. Dicke and E.M. Purcell, "Principles of Microwave Circuits," New York: McGraw-Hill, 1948, chapter 4 , section 22.

[2] J.L. Hesler, "A photonic crystal joint (PCJ) for metal waveguide," 2001 IEEE MTT-S Int. Microwave Symp. Dig., vol. 2, May 2001, pp. 783-786.

[3] R.E. Collins, "Field Theory of Guided Waves," 1960, McGrawHill, chap. 12.

[4] J.L. Hesler, A. R. Kerr, and N. Horner, "A boardband waveguide thermal isolator," Proc. Int. Symp. Space Terahertz Tech. May 2003, vol. 14, pp. 148-154.

[5] J.W. Kooi et. al, "A Full-Height Waveguide to Thin-Film Microstrip Transition with Exceptional RF Bandwidth and Coupling Efficiency", Int J. IR and MM Waves, vol. 24, no. 3, Sep 2003, pp 261-284.

[6] T. Stevenson et. al, "Cosmic Microwave Background Polarization detector with high efficiency, broad bandwidth, and highly symmetric, coupling to transition edge sensor bolometers," J. of Low Temp. Phys, Jan 2008, pp. 471-476.

[7] J.R. Pyle, R.J. Angley, "Cutoff wavelengths of waveguides with unusual cross sections," IEEE Trans. Microwave Theory Tech., vol. 12, no. 5, Sep 1964, pp. 556-557.

[8] Y. S. Chan, C. T. Chan, and Z.Y. Liu, "Photonic band gaps in two dimensional photonic quasicrystals," Phys. Rev. Lett., vol. 80, pp. 956-959, Feb. 1998.

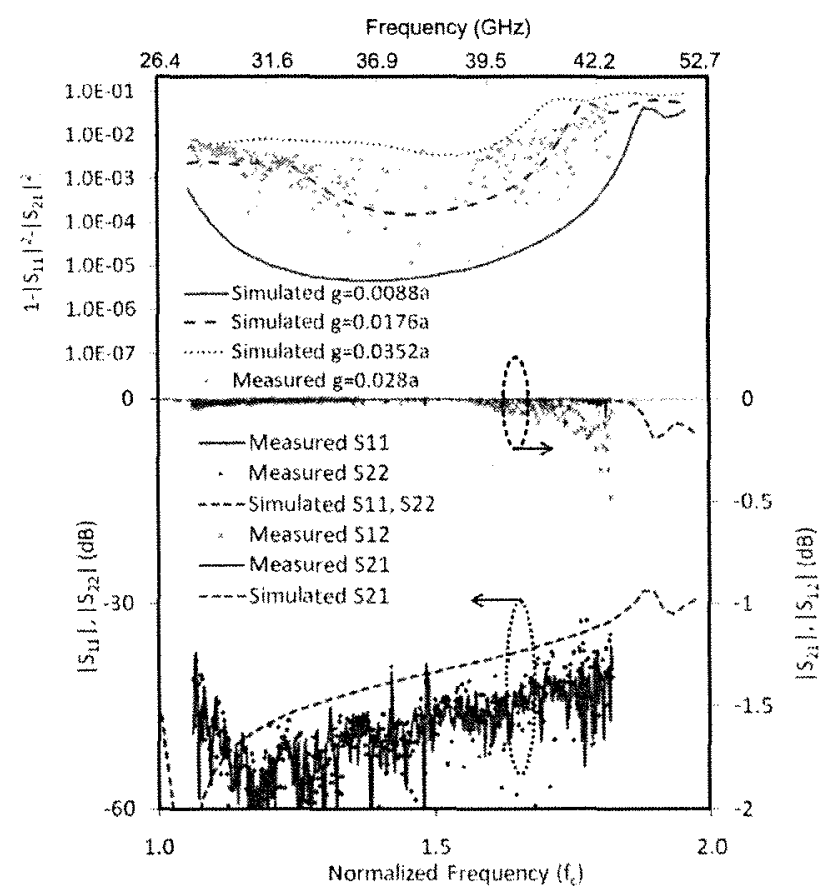

Fig. 9. The frequency response of the measured and simulated $S$ parameters for a Cartesian tiling PCJ is shown. The simulated gap spacings vary from $0.0088 a$ to $0.0352 a$. The measured data corresponds to a gap of $0.028 a$.

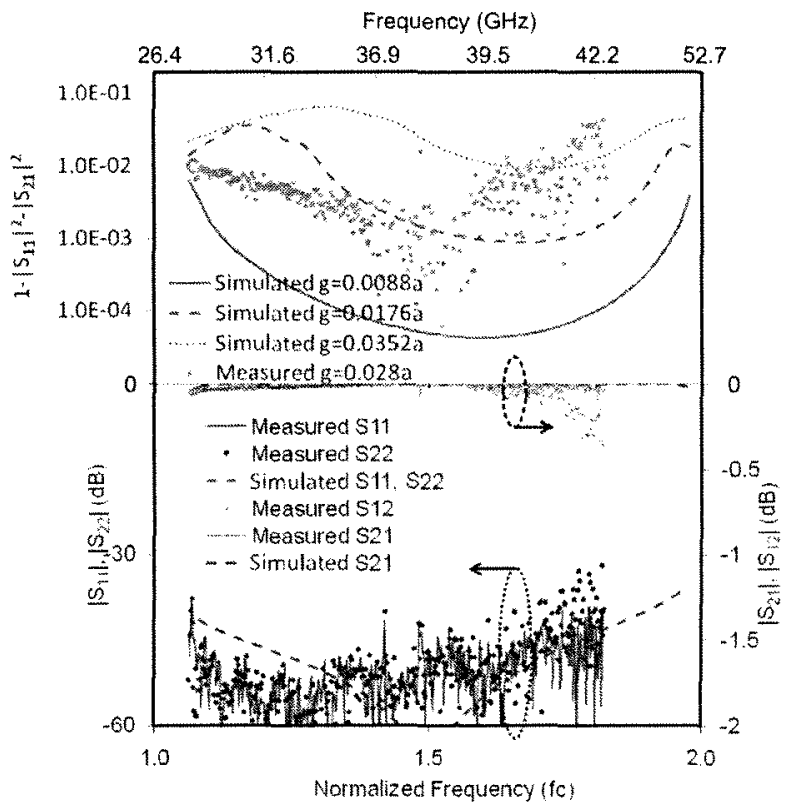

Fig. 10. The frequency responses of the measured and simulated Sparameter for the octagonal tiling PCJ are shown. The simulated gap spacings vary from $0.0088 a$ to $0.0352 a$. The measured data correspond to a gap of $0.028 a$. 